\title{
Faceting and Branching in 2D Crystal Growth
}

\author{
György Tegze, ${ }^{1}$ Gyula I. Tóth, ${ }^{1}$ and László Gránásy ${ }^{1,2}$ \\ ${ }^{1}$ Research Institute for Solid State Physics and Optics, P.O. Box 49, H-1525 Budapest, Hungary \\ ${ }^{2}$ BCAST, Brunel University, Uxbridge, Middlesex, UB8 3PH, United Kingdom
}

(Received 8 November 2010; published 11 May 2011)

\begin{abstract}
Using atomic scale time-dependent density functional calculations we confirm that both diffusioncontrolled and diffusionless crystallization modes exist in simple 2D systems. We provide theoretical evidence that a faceted to nonfaceted transition is coupled to these crystallization modes, and faceting is governed by the local supersaturation at the fluid-crystalline interface. We also show that competing modes of crystallization have a major influence on mesopattern formation. Irregularly branched and porous structures are emerging at the crossover of the crystallization modes. The proposed branching mechanism differs essentially from dendritic fingering driven by diffusive instability.
\end{abstract}

DOI: 10.1103/PhysRevLett.106.195502

PACS numbers: $81.10 . \mathrm{Aj}, 89.75 . \mathrm{Kd}$

A vast variety of natural crystals, including minerals and snowflakes, display flat, smooth surfaces. Faceting is also present in advanced, technologically important materials, such as ceramics [1], semiconductors [2], polymers [3], metallic nanostructures [4], colloid suspensions [5], and monolayers of various surfactant molecules [6,7]. The amazing complexity of faceted patterns has been generating considerable interest for decades. Various crystal shapes; compact symmetric, dendritic, ramified fractal and needlelike morphologies have been investigated experimentally [1-5]. While robust theoretical interpretations of faceted equilibrium shapes exist, the dynamical aspects of faceted pattern formation are less understood. The main difficulty in establishing a theoretical description lies in the multiscale nature of faceted crystal growth, the mesoscale behavior is influenced by the local arrangement of the particles. Russel et al. [8] have identified some mesoscale characteristics of crystal growth kinetics: (i) a diffusion-controlled (or "slow") growth mode that may lead to diffusive instability and dendritic branching in monodisperse colloids [8,9], and (ii) a diffusionless (or "fast") steady growth mode, in which crystalline ordering takes place without significant density change. The existence of these modes is verified experimentally by investigating crystallization kinetics in hard-sphere suspensions $[10,11]$. Besides, in some organic glasses discontinuous enhancement of crystal growth is observed [12-14], which is associated with diffusionless crystallization [15]. Although various theories have been developed to explain the existence of diffusion-controlled and diffusionless rapid modes [14-16], none of them predicts a facetednonfaceted morphological transition often associated with different crystallization modes, when interpreting experiments [3]. Growth mode (i) and mode (ii) have also been observed in simple mean-field models [17], although these theories address anisotropic crystal growth in a phenomenological way. In contrast, molecular theories based on the classical density functional technique (DFT) predict not only the anisotropic bulk crystal properties, such as the crystal structure and elastic constants, but also anisotropic surface properties (e.g., surface tension [18]). A simple dynamical DFT [19], the phase-field crystal (PFC) model [20] is able to address crystallization kinetics up to the mesoscale [21]. While the phase-field crystal model represents the average of the microscopic states over a coarse graining time, it does describe crystal defects. Grain boundaries and dislocations can be observed directly in simulations [20], while diffusing point defects (i.e., vacancies) are time averaged and are represented by a lower filling factor (lower integral density) at the lattice sites [22]. The Kirkendall effect that assumes vacancy diffusion has also been investigated in the binary PFC model [23]. It has also been shown that the crystal growth anisotropy is inherent in the PFC model [24].

In this Letter we investigate faceting during crystal growth using the PFC model, and interpret crystalline pattern formation in terms of two basic crystal growth mechanisms [type (i) and (ii)]. Although we simulated surface diffusion-coupled 2D crystallization (relevant directly for $2 \mathrm{D}$ colloids, surfactant monolayers and island formation on substrates, etc.), the conclusions we are going to draw are more generic. None of the underlying processes (e.g., faceting, and diffusion-controlled growth) are specific to these systems.

The PFC model we use relies on the time-averaged particle density $\rho$, and applies on the diffusional time scale. The dimensionless free energy of the inhomogeneous system relative to a homogeneous reference state of density $\rho_{L}^{\text {ref }}$ can be derived [25] from the Ramakrishnan-Yussouff type DFT [26]:

$$
\mathcal{F}=\int d \mathbf{r}\left\{\frac{\psi}{2}\left[\epsilon+\left(1+\nabla^{2}\right)^{2}\right] \psi+\frac{\psi^{4}}{4}\right\},
$$

where $\psi \propto\left(\rho-\rho_{L}^{\text {ref }}\right) / \rho_{L}^{\text {ref }}$ is the scaled density difference relative to $\rho_{L}^{\text {ref }}$. The model parameter $\epsilon$ can be connected to such physical properties as the bulk moduli of the fluid and 
crystalline phases at the reference density and temperature. In two dimensions, the homogeneous fluid and the hexagonal crystal can coexist [20]. The transition layer between these coexisting phases can be either thin or several atomic layers thick. The interface thickness is tuned by the model parameter $\epsilon$. Crystallization and the diffusional transport are described by the time evolution of the particle density $(\psi)$, and governed by a diffusive equation of motion:

$$
\frac{\partial \psi}{\partial \tau}=\nabla^{2} \frac{\delta \mathcal{F}}{\delta \psi}+\zeta
$$

where $\tau$ is the dimensionless time, and $\zeta$ represents a colored Gaussian noise characterized by the correlator $\left\langle\zeta(\mathbf{r}, \tau) \zeta\left(\mathbf{r}^{\prime}, \tau^{\prime}\right)\right\rangle=-\alpha \nabla^{2} g\left(\left|\mathbf{r}-\mathbf{r}^{\prime}\right|, \lambda\right) \delta\left(\tau-\tau^{\prime}\right)$. Here $\alpha$ controls the magnitude of the thermal fluctuations relative to the free energy barriers and $g\left(\left|\mathbf{r}-\mathbf{r}^{\prime}\right| / \lambda\right)$ is a high frequency cutoff function [27] for wavelengths shorter than the lattice spacing $(\lambda)$.

First, we investigate the dynamic properties of the crystal-fluid interface as a function of the thermodynamic driving force. For the sake of simplicity, we set the noise correlator to zero $(\alpha=0)$, and choose $\epsilon=-0.75$ to ensure faceted equilibrium shape. Crystal growth has been initiated using a crystal seed, while the initial particle density $\left(\psi_{0}\right)$ of the fluid phase has been used to tune the thermodynamic driving force of the phase transition.

Some of the crystallization kinetics dependent interface properties are summarized in Fig. 1. At low crystal growth rate, the interface is fairly thin and faceted [Fig. 1(a)], while at high growth rate it extends to several atomic layers and shows rounded corners [Fig. 1(b)]. Dynamical reduction of the growth anisotropy has also been observed in molecular monolayers and proved to be mainly of kinetic origin [28].

The time evolution of the coarse-grained particle density $(\tilde{\psi})$ profile shows distinct characteristics for faceted and nonfaceted interfaces. ( $\tilde{\psi}$ has been obtained by twodimensional finite impulse response filtering [29].) At low driving forces the time dependence of the interface velocity is parabolic $\left(v \propto \tau^{-1 / 2}\right)$. Crystal growth is controlled by diffusive transport through an extending depletion layer [growth mode (i), see Fig. 1(c)]. At high supersaturations crystal growth is limited by the rate of particle rearrangement at the interface from liquid state to solid state [growth mode (ii)]: here the diffusion layer shows a steady profile [Fig. 1(d)] and the interface velocity is constant. As opposed to growth mode (i), the density difference between the crystalline and liquid phases is substantially reduced, which can be interpreted as "vacancy trapping" into the crystal, analogously to the phenomenon of "solute trapping" in rapidly solidifying alloys. The concentration of the quenched-in vacancies [30] can be characterized by the average crystal density. Following Aziz [31], we have defined an effective partition
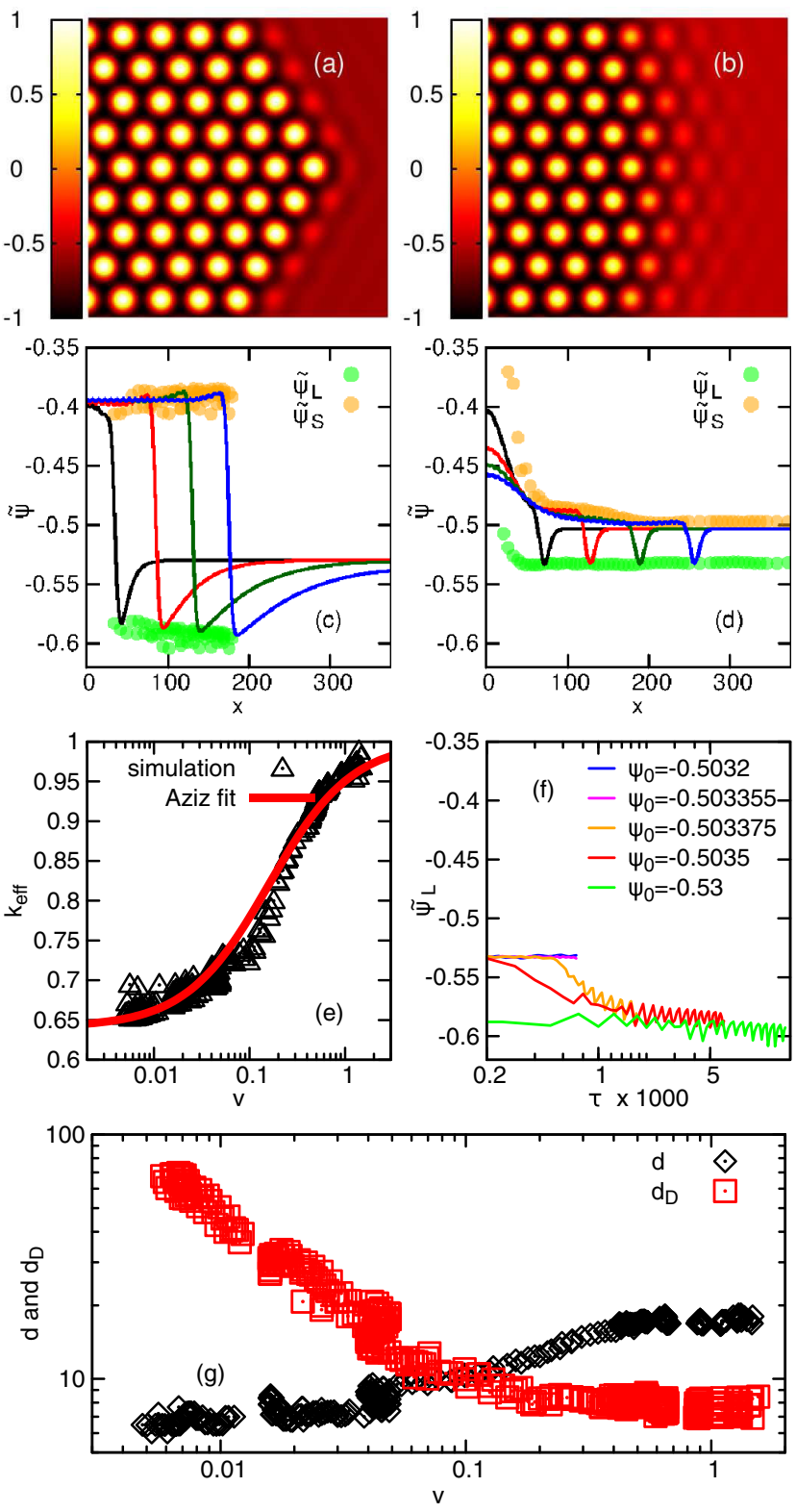

FIG. 1 (color online). Two modes of interface propagation: Close-up view of the density map at the interface for the (a) diffusion-controlled "slow" $\left(\psi_{0}=-0.53\right)$ (b) diffusionless "fast" ( $\left.\psi_{0}=-0.5032\right)$ growth modes. The coarse-grained density profiles $(\tilde{\psi})$ are shown at different times for (c) the slow $\left(\psi_{0}=-0.53\right)$ and $(\mathrm{d})$ the fast $\left(\psi_{0}=-0.5032\right)$ modes. Minima of the curves correspond to the fluid density at the interface $\left(\tilde{\psi}_{L}\right)$, while the density steps left to the minima represent the density change across the fluid-crystalline interface. $\tilde{\psi}_{S}$ is the solid density at the interface. (e)-(g) Merged data for $\psi_{0}=$ $-0.53 ;-0.5035 ;-0.503375 ;-0.50335 ;-0.5032$ are plotted: (e) $k_{\text {eff }}$ vs velocity. (f) $\tilde{\psi}_{L}$ vs time. (g) The interface thickness, $d$, and diffusion length, $d_{D}$ vs velocity.

coefficient using the average solid and liquid densities at the interface as $k_{\text {eff }}=\tilde{\psi}_{S} / \tilde{\psi}_{L}$ and we have fitted $k_{\text {eff }}=k_{0}^{1 /\left(1+v / v_{D}\right)}$ to our data. In the formula $k_{0}=$ $\tilde{\psi}_{S}^{E} / \tilde{\psi}_{L}^{E}=0.649$ is the equilibrium partition coefficient 
defined by the phase diagram of the system [20], $v$ is the interface velocity, while $v_{D}$ is the fitted characteristic interface velocity for point-defect trapping. The effective partition coefficient vs interface velocity data are merged into master plots [Fig. 1(e)] for a sequence of simulations $\left(\psi_{0}=-0.53 ;-0.5035 ;-0.503375 ;-0.50335\right.$ and -0.5032 ). This master plot shows that the solid-liquid density difference at the interface decreases as the growth rate increases $\left(k_{\text {eff }}\right.$ increases towards 1 at high interface velocities). The density of the crystalline phase is dynamically adjusted to the fluid density, making steady growth feasible. The Aziz formula is in convincing agreement with our data which implies that the density change corresponds to defect trapping. Similar behavior has been observed in simple field theoretic models [32].

While at first glance mode switching in the dynamics is hidden, the faceted to nonfaceted transition associated with this phenomenon is quite spectacular. A microscopic explanation of faceting can be given considering the local thermodynamic conditions at the interface. Close to equilibrium, in the faceted growth regime, the crystal grows layerwise; filling of a lattice site in an existing crystal layer is preferred to new layer formation via nucleation. At the interface $\tilde{\psi}_{L}$ oscillates in time [Fig. 1(f)], where each breakdown from a local maximum corresponds to the nucleation of a crystal layer. Figure 1(f) also shows that increasing $\tilde{\psi}_{L}$ at the interface results in a vanishing barrier for crystal layer nucleation, the interface becomes rough on the atomic scale, and as a consequence, faceted to nonfaceted transition occurs. Also note that for layer-by-layer growth vacancy trapping is hardly feasible since the filling of sites in the existing layers is preferred to new layer formation.

Besides faceting, surface roughening [33] is manifested in dynamical interface broadening [34] as the growth rate increases [see Fig. 1(g)]. Figure $1(\mathrm{~g})$ also shows that the interface thickness crosses the diffusion length as the interface velocity increases. The mode switching occurs at the crossover of these curves, at high driving forces the interface layer and the depleted zone overlap, due to trapping of vacancies into the interface layer.

Next, we examine mesoscale patterning near mode switching, and compare the simulations to micrographs of 2D colloid crystals. The free growth of single crystals has been investigated in the presence of thermal fluctuations $\left(\alpha=4 \times 10^{-3}\right)$. In the diffusion-controlled regime faceted dendritic mesopatterns evolve [Fig. 2(c)]; branching is driven by diffusive instability [35]. At higher supersaturations thermal fluctuation driven random switching from fast to slow mode is observed along the interface. The coexisting slow and fast mode interfaces outline fractal-like mesopatterns [Figs. 2(d) and 2(e)]. Contrary to diffusive instability driven branching, fractal-like arms are due to the difference in the propagation velocity for slow and fast mode interfaces. Pore formation via consecu-
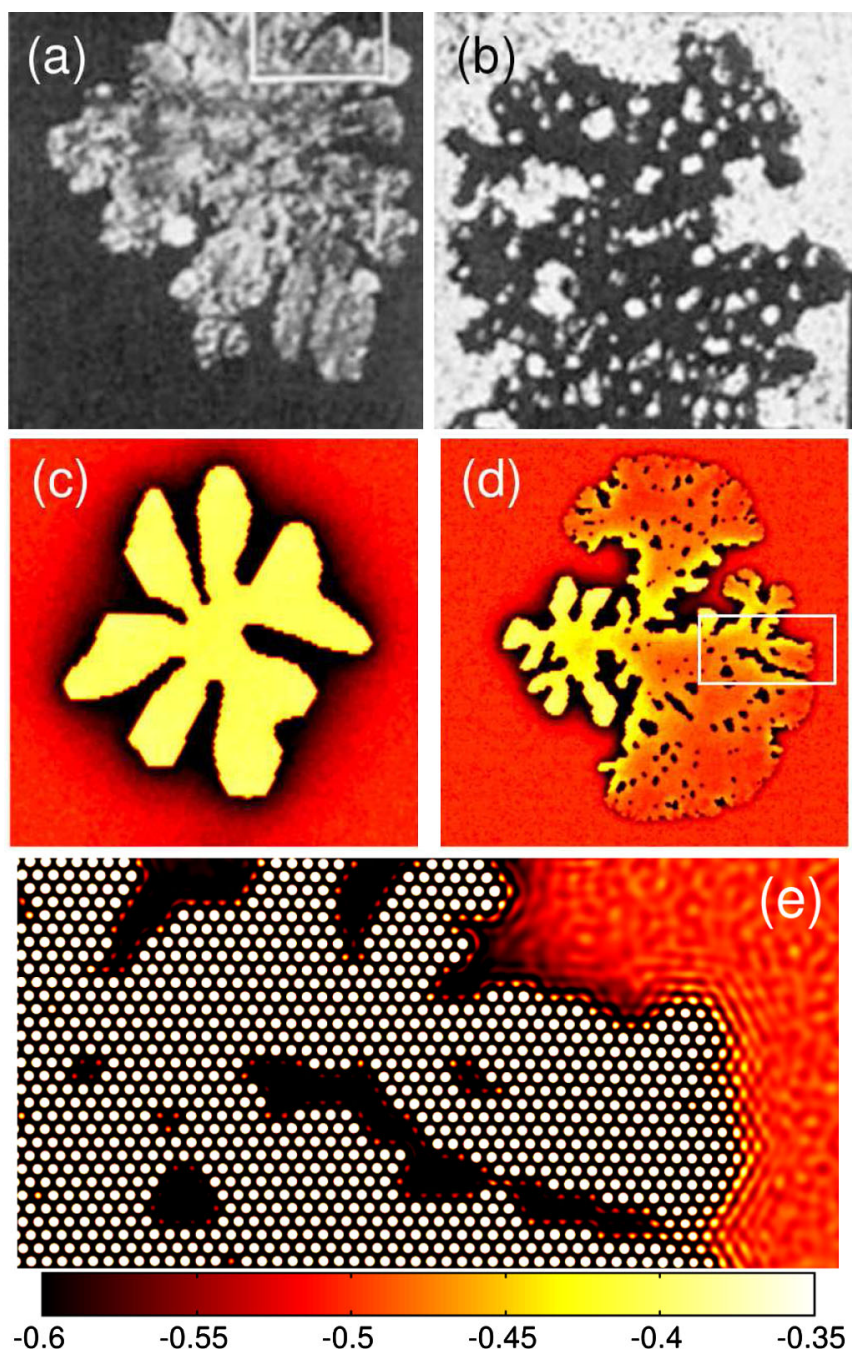

FIG. 2 (color online). Single crystal growth patterns in the presence of thermal fluctuations: (a),(b): dendritic and fractallike 2D colloid crystals (micrographs by Skjeltorp [5]). (c),(d): Coarse-grained density maps for dendritic $\left(\psi_{0}=-0.505\right)$ and fractal-like branched patterns $\left(\psi_{0}=-0.504\right)$ in PFC simulations. (e) Fractal branching mechanism: Close-up view of modeswitching along the interface. Density waves in the liquid inherent to the fast mode, while depleted layer (dark) indicates slow mode interface propagation.

tive branching and merging of fractal arms has also been observed [see Fig. 2(e)], which is not common when branching is driven by diffusive instability. Such Swisscheese-like patterns are often observed in monolayers $[5,36]$. Simulations show a striking resemblance to morphologies observed in optical micrographs of colloid single crystals [5] [see Fig. 2(a) and 2(b)], and fractal-like growth in molecular monolayers [28].

We note that the fast and slow modes can coexist even in the zero noise limit $(\alpha=0)$. In this case, vacancy trapping and mode selection are governed by the local atomic arrangement at the solid-liquid interface that varies with crystallographic orientation. Increasing the noise 
amplitude, the fast mode becomes increasingly preferred, while branching events become more frequent and the porosity of the growing crystal increases.

Summarizing, using a microscopic theory, symbiosis of faceting with growth mode selection is predicted: if faceted growth exists at a temperature $(\epsilon)$, the slow mode, which exhibits decreasing supersaturation necessarily leads to layer-by-layer growth. Also layerwise growth largely prevents excess vacancy trapping into the crystal; thus, the dynamical adjustment of crystal density and the "fast" mode interface propagation are not feasible. We have investigated crystallization kinetics dependent interface properties: the dynamical reduction of crystal growth anisotropy and interface broadening shown are in agreement with previous reports [34]. Ultimately, we have shown that the slow and the fast propagation modes can coexist along the interface of the crystal. The thermal fluctuation driven random mode switching leads to a new branching mechanism that explains irregular fractal-like growth of single crystals for the first time.

This work has been supported by the EU FP7 Collaborative Project ENSEMBLE under Grant Agreement NMP4-SL-2008-213669 and by the Hungarian Academy of Sciences under Contract No. OTKA-K-62588.

[1] Z. Quian, T. L. Tolt, and A. R. Cooper, J. Am. Ceram. Soc. 70, 48 (1987).

[2] Q. Wang et al., Scr. Mater. 54, 37 (2006).

[3] G. Zhang et al., J. Chem. Phys. 129, 224708 (2008).

[4] Y. Zhu et al., Mater. Res. Bull. 38, 1829 (2003).

[5] A. T. Skjeltorp, Phys. Rev. Lett. 58, 1444 (1987).

[6] M. Wang et al., Phys. Rev. Lett. 71, 4003 (1993).

[7] B. Berge, L. Faucheux, K. Schwab, and A. Libchaber, Nature (London) 350, 322 (1991).

[8] W. B. Russel, P. M. Chaikin, J. Zhu, W. V. Meyer, and R. Rogers, Langmuir 13, 3871 (1997).

[9] Z. Cheng, P. M. Chaikin, J. Zhu, W. B. Russel, and W. V. Meyer, Phys. Rev. Lett. 88, 015501 (2001).

[10] Y. He, B. J. Ackerson, W. van Megen, S. M. Underwood, and K. Schätzel, Phys. Rev. E 54, 5286 (1996).

[11] D. J. W. Aastuen, N.A. Clark, L. K. Cotter, and B. J. Ackerson, Phys. Rev. Lett. 57, 1733 (1986).
[12] T. Hikima, M. Hanaya, and M. Oguni, J. Mol. Struct. 479, 245 (1999).

[13] M. Hatase, M. Hanaya, and M. Oguni, J. Non-Cryst. Solids 333, 129 (2004).

[14] Y. Sun, H. Xi, M. D. Ediger, and L. Yu, J. Phys. Chem. B 112, 661 (2008).

[15] T. Konishi and H. Tanaka, Phys. Rev. B 76, 220201 (2007).

[16] T. Hikima, Y. Adachi, M. Hanaya, and M. Oguni, Phys. Rev. B 52, 3900 (1995).

[17] R. Wild and P. Harrowell, J. Chem. Phys. 114, 9059 (2001).

[18] P. Harrowell and D. W. Oxtoby, Phys. Rev. B 33, 6293 (1986).

[19] S. van Teeffelen, C. N. Likos, and H. Löwen, Phys. Rev. Lett. 100, 108302 (2008).

[20] K. R. Elder, M. Katakowski, M. Haataja, and M. Grant, Phys. Rev. Lett. 88, 245701 (2002).

[21] T. Pusztai, G. Tegze, G. I. Tóth, L. Környei, G. Bansel, Z. Fan, and L. Gránásy, J. Phys. Condens. Matter 20, 404205 (2008).

[22] K. R. Elder and M. Grant, Phys. Rev. E 70, 051605 (2004).

[23] K. R. Elder, K. Thornton, and J. J. Hoyt, Philos. Mag. 91, 151 (2011).

[24] G. Tegze et al., Phys. Rev. Lett. 103, 035702 (2009).

[25] K. R. Elder, N. Provatas, J. Berry, P. Stefanovic, and M. Grant, Phys. Rev. B 75, 064107 (2007).

[26] T. V. Ramakrishnan and M. Yussouff, Phys. Rev. B 19, 2775 (1979).

[27] J. G. Ojalvo and J. M. Sancho, Noise in Spatially Extended Systems (Springer, New York, 1999), p. 175.

[28] J. Flesselles, M. O. Magnasco, and A. Libchaber, Phys. Rev. Lett. 67, 2489 (1991).

[29] R. L. Davidchack and B. B. Laird, J. Chem. Phys. 108, 9452 (1998).

[30] Y. Yang and I. Baker, Intermetallics 6, 167 (1998).

[31] M. J. Aziz, J. Appl. Phys. 53, 1158 (1982).

[32] H. Löwen, J. Bechhoefer, and L. S. Tuckerman, Phys. Rev. A 45, 2399 (1992).

[33] E. Brückner and D. Stauffer, Z. Phys. 53, 241 (1983).

[34] R. P. A. Dullens, D. G. A. L. Aarts, and W. K. Kegel, Phys. Rev. Lett. 97, 228301 (2006).

[35] W. W. Mullins and R. F. Sekerka, J. Appl. Phys. 35, 444 (1964).

[36] D. K. Schwartz, S. Steinberg, J. Israelachvili, and J. A. N. Zasadzinski, Phys. Rev. Lett. 69, 3354 (1992). 